\title{
Insulin resistance and clinical outcomes after acute ischemic stroke
}
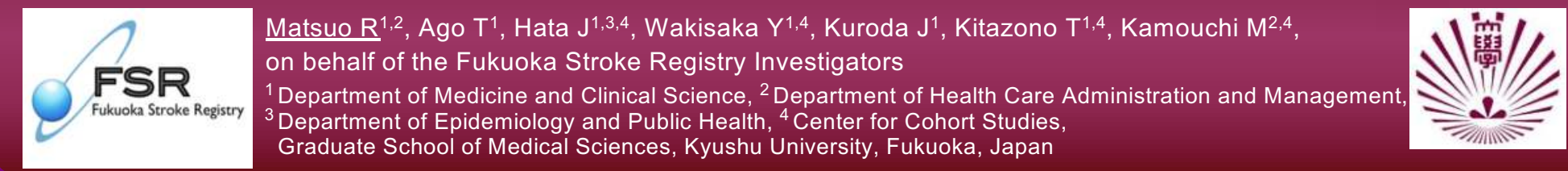

Introduction \& Objective

Poor glycemic control before stroke onset was associated with unfavorable functional outcomes independent of blood glucose levels in patients with acute ischemic stroke. However, it is unknown which mechanisms contribute to this association. This study aimed to determine whether homeostasis model assessment of insulin resistance (HOMA-IR), a marker for insulin resistance, is associated with clinical outcomes after acute ischemic stroke.

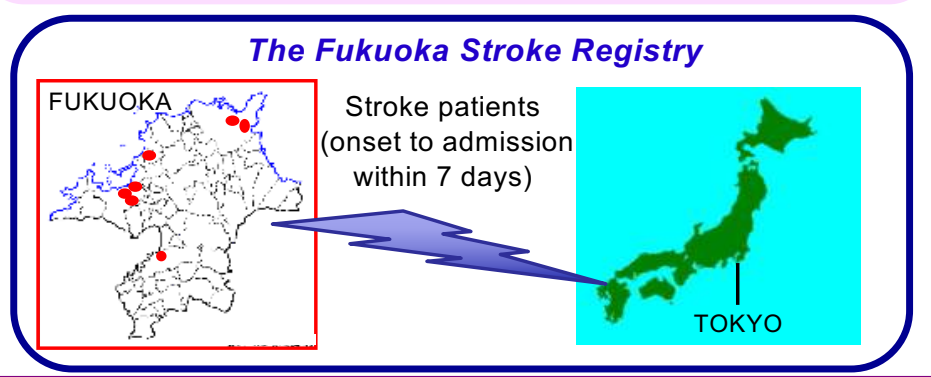

\section{Methods}

<Participants>

Participants were $\mathbf{4 6 5 5}$ patients with acute ischemic stroke (age 70.3 \pm 12.5 years; $63.5 \%$ men) who had been independent before admission, were registered from April 2009 to March 2015, and did not receive insulin therapy during hospitalization.

$<$ HOMA-IR score $>$ fasting glucose $(\mathrm{mU} / \mathrm{L}) \times$ fasting insulin $(\mathrm{mg} / \mathrm{dl})$

<Study outcomes>

405

\section{Neurologic improvement:}

$\geq 4$-point decrease in the NIHSS score during hospitalization or an NIHSS score of 0 at discharge.

\section{Neurologic worsening}

$\geq 2$-point increase in NIHSS score hospitalization compared with the NIHSS score on admission.

\section{Poor functional outcome at $\mathbf{3}$ months:}

functional dependency (mRS score of 3-5) or death (mRS score of 6) at 3 months.

\section{Results}

Table. Baseline characteristics of patients according to HOMA-IR score

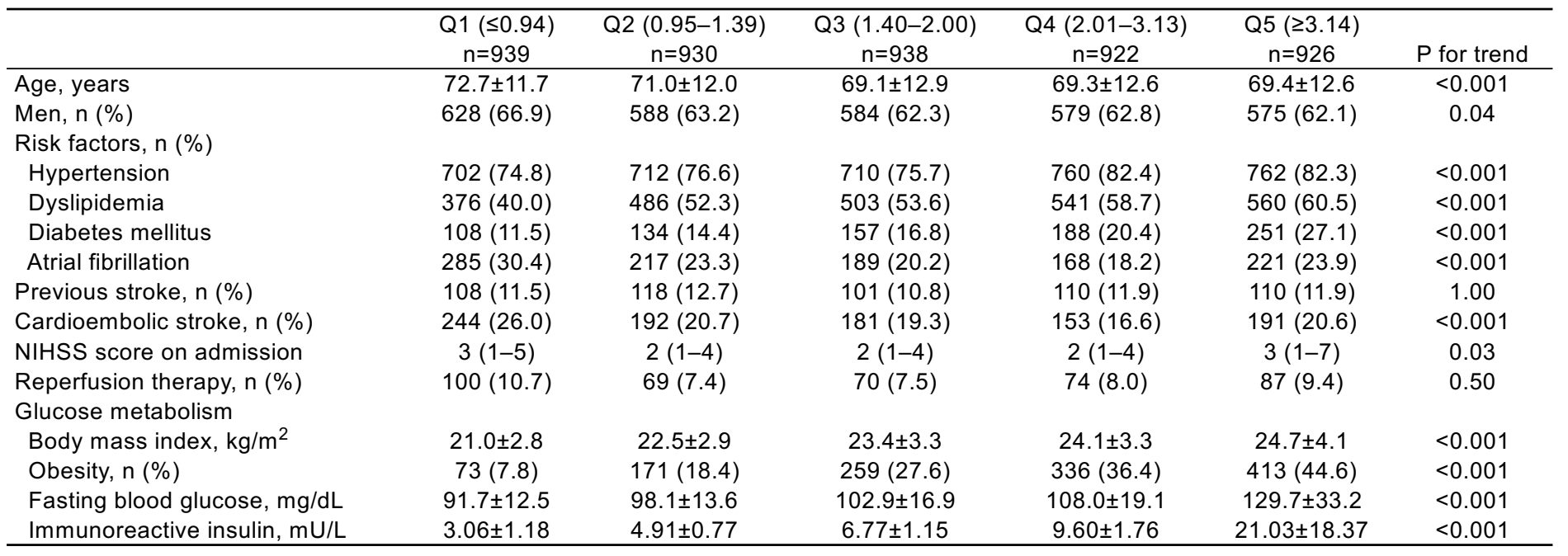

Figure. HOMA-IR score and clinical outcomes

\begin{tabular}{|c|c|c|c|c|c|c|}
\hline \multirow{6}{*}{$\begin{array}{r}\text { Neurologic } \\
\text { improvement }\end{array}$} & & Events (\%) & \multirow{2}{*}{$\begin{array}{r}\text { OR } \\
\vdots\end{array}$} & OR & $95 \% \mathrm{Cl}$ & $\mathrm{P}$ \\
\hline & Q1 & $572(60.9)$ & & 1.00 & (reference) & \\
\hline & Q2 & $570(61.3)$ & & 0.98 & $(0.81-1.19)$ & 0.84 \\
\hline & Q3 & $555(59.2)$ & & 0.87 & $(0.71-1.05)$ & 0.14 \\
\hline & Q4 & $523(56.7)$ & & 0.77 & $(0.63-0.94)$ & 0.009 \\
\hline & Q5 & $494(53.4)$ & & 0.68 & $(0.56-0.83)$ & $<0.001$ \\
\hline \multirow{5}{*}{$\begin{array}{r}\text { Neurologic } \\
\text { worsening }\end{array}$} & Q1 & $62(6.6)$ & & 1.00 & (reference) & \\
\hline & Q2 & $39(4.2)$ & & 0.65 & $(0.43-0.99)$ & 0.046 \\
\hline & Q3 & $55(5.9)$ & & 0.98 & $(0.67-1.44)$ & 0.92 \\
\hline & Q4 & $59(6.4)$ & & 1.08 & $(0.73-1.59)$ & 0.71 \\
\hline & Q5 & $98(10.6)$ & & 1.86 & $(1.30-2.67)$ & 0.001 \\
\hline \multirow{6}{*}{$\begin{array}{r}\text { Poor } \\
\text { functional } \\
\text { outcome }\end{array}$} & Q1 & $203(21.6)$ & & 1.00 & (reference) & \\
\hline & Q2 & $152(16.3)$ & & 0.87 & $(0.65-1.16)$ & 0.34 \\
\hline & Q3 & $154(16.4)$ & & 0.97 & $(0.72-1.29)$ & 0.82 \\
\hline & Q4 & $167(18.1)$ & & 1.21 & $(0.90-1.61)$ & 0.21 \\
\hline & Q5 & $280(30.2)$ & & 2.02 & $(1.52-2.68)$ & $<0.001$ \\
\hline & & 0.3 & 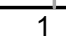 & & & \\
\hline
\end{tabular}

Multivariables: age, sex, reperfusion therapy, NIHSS score on admission, stroke subtype (cardioembolic stroke), diabetes status, and body mass index.

Discussion

\section{Insulin resistance}

- prevent recovery from the ischemic insult and subsequently worsens the functional outcome after acute ischemic stroke.

$\checkmark$ Pro-inflammatory cytokines may enhance local inflammatory and prothrombotic responses.

$\checkmark$ Insulin resistance may lead to impaired circulation in the ischemic penumbra.

$\checkmark$ Insufficient insulin action can cause catabolism in muscles, including paralyzed muscles.

\section{Conclusion}

Insulin resistance is associated with poor

functional outcome in patients with acute ischemic stroke. These findings shed new light on insulin resistance as a therapeutic target in improving functional outcomes after acute ischemic stroke. 